\title{
FORM DRAG AND EQUIVALENT SAND-GRAIN ROUGHNESS FOR WAVE-GENERATED SAND RIPPLES
}

\author{
Jing Yuan, National University of Singapore, ceeyuan@nus.edu.sg \\ Dongxu Wang, National University of Singapore, wang_dongxu@u.nus.edu
}

\begin{abstract}
Moderate shoaling waves usually generate some smallscale bottom bedforms, i.e. sand ripples, which are 1$10 \mathrm{~cm}$ tall and $10-100 \mathrm{~cm}$ long. Wave-induced boundary layer flows over sand ripples are characterized by coherent vortices, which are alternatively generated on both sizes of ripples under the oscillatory free-stream motion. This process leads to a form drag, which significantly increases the local flow resistance. A large equivalent sand-grain roughness scaled with ripple dimension is therefore adopted in coastal models to account for ripple presences. Very few quantitative experimental results on this topic are available in the literature, so this study is aimed at filling this gap.
\end{abstract}

Experiments are conducted in the newly developed oscillatory water tunnel (OWT) at the hydraulic lab at the National University of Singapore. This facility is essentially a U-tube with an enclosed horizontal test section, so a piston located at one end of the OWT can easily produce oscillatory flows corresponding to fullscale conditions in its $10 \mathrm{~m}$-long test section (Yuan and Madsen 2014). To directly measure the form drag for oscillatory flows over sand ripples, a novel pressurebased technique is developed as follows. As shown in Figure 1, the oscillatory flow in the test section is driven by a pressure difference between the two ends, i.e. $p(t)$ $p_{0}(t)$. Under the same piston motion, the pressure at the open-riser end, $p_{0}(t)$, will remain the same, but the pressure at the piston end, $p(t)$, will vary with the flow resistance in the test section. Comparing two tests with identical piston oscillatory motions but different bed conditions, i.e. one with a flat bed and the other with a rippled bed, the difference in $p(t)$ is solely related to the ripple-induced form drag. Thus, a direct measurement of form-drag shear stress can be obtained. The total flow resistance can be estimated by adding a prediction of skin-friction shear stress. Three pressure transducers are installed near the bottom of the piston-end cylindrical riser (as indicated in Figure 1). Measurements suggest a very good agreement among the three transducers and a very good repeatability of the experiments. A laser-based bottom profiler has been developed to capture ripple geometry in the test section.

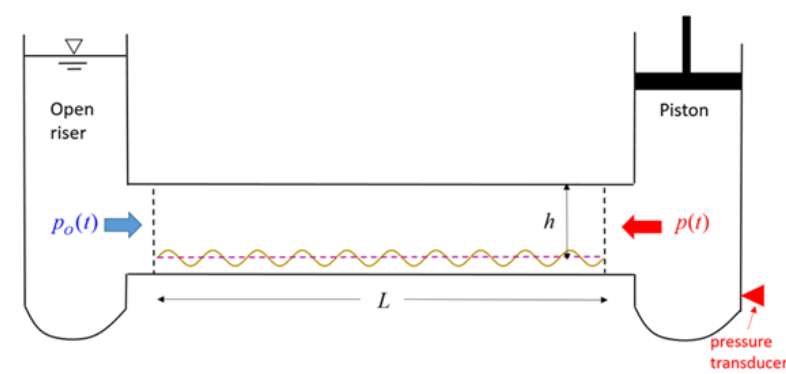

Figure 1 Sketch of the OWT and the concept of the pressure-based technique for measuring form drag

In this study, tests are conducted with sinusoidal oscillatory flows over fully-developed sand ripples. The amplitude $U_{b}$ and period $T$ of the free-stream flow are from $0.3 \mathrm{~m} / \mathrm{s} \sim 0.8 \mathrm{~m} / \mathrm{s}$ and $4.17 \mathrm{~s} \sim 8 \mathrm{~s}$, respectively. The movable bed is made of coarse sand with a medium diameter of $0.51 \mathrm{~mm}$. Some post-processing is required to filter out high-frequency noise in pressure measurements, which is due to the facility resonance. Figure 2 shows the normalized form-drag shear stress for a typical test with $U_{b}=0.4 \mathrm{~m} / \mathrm{s}$ and $T=6.25 \mathrm{~s}$. The ripple height and length are $92 \mathrm{~mm}$ and $456 \mathrm{~mm}$, respectively. It can be seen that the form drag varies almost in-phase with the free-stream velocity with a slight phase lag. Its normalized magnitude is up to 0.15 . which is much larger than the skin-friction shear stress (of order 0.01), indicating the dominance of form drag in the ripple-bed regime.

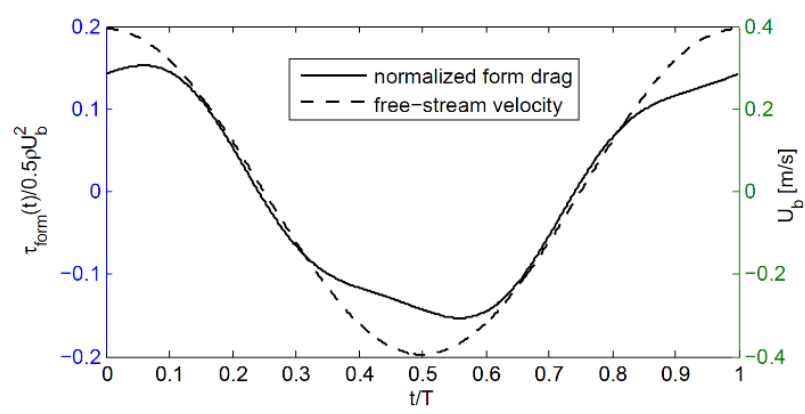

Figure 2 intra-period variation of normalized form drag

The amplitude of dominant first-harmonic total shear stress is further converted to a wave-friction factor and compared with the model predictions with an equivalent sand-grain roughness suggested by Madsen (2002), i.e. $k_{b}=4 \eta$, where $\eta$ is the measured ripple height. As shown in Figure 3, the model, which is derived based on a smallroughness assumption, can reasonably estimate the amplitude of total shear stress for sand ripples. However, the model predicts that the maximum bottom shear stress leads the free-stream flow by $20-45^{\circ}$ in phase, while observation suggests a phase lag. This indicates that simple wave boundary layer models derived based on the assumption of a small roughness must be corrected for phase prediction.

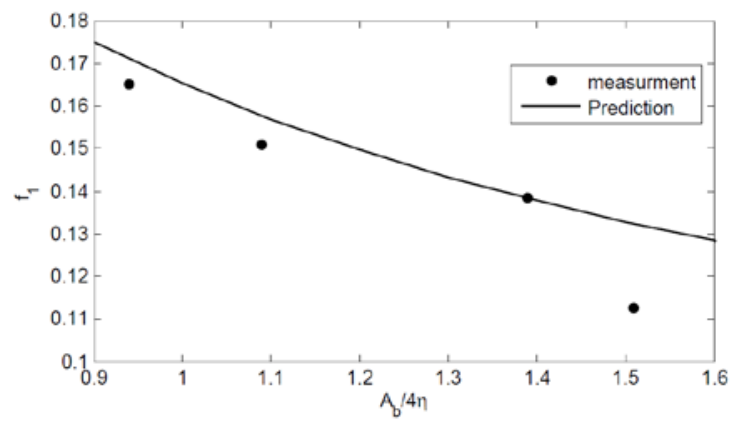

Figure 1 first-harmonic friction factor

\section{REFERENCES}

Yuan, J. and Madsen, O.S. (2014), Experimental study of turbulent oscillatory boundary layers in an oscillating water tunnel. Coastal Engineering, 89: 63-84.

Madsen, O. S. (2002), Sediment transport outside the surf zone, Coastal Engineering Manual, vol. III. U.S. Army Corps of Engineers, Washington DC. Chapter 6. 